

\title{
CLASSIFICATION OF POSITIVE SINGULAR SOLUTIONS TO A NONLINEAR BIHARMONIC EQUATION WITH CRITICAL EXPONENT
}

\author{
RUPERT L. FRANK AND TOBIAS KÖNIG
}

For $n \geq 5$, we consider positive solutions $u$ of the biharmonic equation

$$
\Delta^{2} u=u^{(n+4) /(n-4)} \quad \text { on } \mathbb{R}^{n} \backslash\{0\},
$$

with a nonremovable singularity at the origin. We show that $|x|^{(n-4) / 2} u$ is a periodic function of $\ln |x|$ and we classify all periodic functions obtained in this way. This result is relevant for the description of the asymptotic behavior of local solutions near singularities and for the $Q$-curvature problem in conformal geometry.

\section{Introduction and main results}

In this paper we are interested in positive solutions $u$ of the equation

$$
\Delta^{2} u=u^{(n+4) /(n-4)} \quad \text { in } \mathbb{R}^{n} \backslash\{0\}
$$

for $n \geq 5$. As we will explain later in more detail, this equation serves on one hand as a model problem for higher-order equations with critical nonlinearity and on the other hand has a concrete meaning in the $Q$-curvature problem in conformal geometry. It is well known that the absence of the maximum principle for equations involving the bi-Laplacian poses great challenges both on a conceptual and on a technical level. Nevertheless we succeed here in proving a classification result for positive solutions of (1) which is completely analogous to its second-order counterpart.

We will work throughout with classical solutions of (1), that is, $u \in C^{4}\left(\mathbb{R}^{n} \backslash\{0\}\right)$. Because of the regularity theory in [Uhlenbeck and Viaclovsky 2000] (which extends that in [Chang et al. 1999] to $n \geq 5$ ) this is not a restriction.

In the fundamental work [Lin 1998] it was shown that all solutions $u$ with a removable singularity at the origin (so that (1) holds in all of $\mathbb{R}^{n}$ ) are given by

$$
u(x)=c_{n}\left(\frac{\lambda}{1+\lambda^{2}\left|x-x_{0}\right|^{2}}\right)^{(n-4) / 2}, \quad c_{n}=((n-4)(n-2) n(n+2))^{(n-4) / 8},
$$

for some $\lambda>0$ and $x_{0} \in \mathbb{R}^{n}$. Solutions of the closely related equation $\Delta^{2} u=|u|^{8 /(n-4)} u$ in $\mathbb{R}^{n}$ are, in particular, given by optimizers of the Sobolev inequality

$$
\int_{\mathbb{R}^{n}}(\Delta u)^{2} \mathrm{~d} x \geq \mathcal{S}_{n}\left(\int_{\mathbb{R}^{n}}|u|^{2 n /(n-4)} \mathrm{d} x\right)^{(n-4) / n} .
$$

Frank was partially supported through the National Science Foundation grant DMS-1363432 .

MSC2010: 34C25, 35B09, 35J30, 53A30.

Keywords: fourth-order equation, critical exponent, classification, periodic solutions. 
These optimizers were classified in [Lieb 1983] in an equivalent dual formulation and are again given by constant multiples of the functions in (2). For a classification of positive solutions with removable singularities of the four-dimensional analogue of (1) we refer to [Chang and Yang 1997; Lin 1998] and for the higher-order case to [Wei and Xu 1999; Martinazzi 2009].

In this paper we will be concerned with solutions $u$ of (1) with nonremovable singularities. It was also shown by Lin [1998] that such solutions are necessarily radial. We pass to logarithmic coordinates (in this context also known as Emden-Fowler coordinates) and write

$$
u(x)=|x|^{-(n-4) / 2} v(\ln |x|) .
$$

By a short computation we find that (1) for $u$ is equivalent to the following ordinary differential equation for $v$ :

$$
v^{(4)}-\frac{n(n-4)+8}{2} v^{\prime \prime}+\frac{n^{2}(n-4)^{2}}{16} v-|v|^{8 /(n-4)} v=0 \quad \text { in } \mathbb{R} .
$$

Note that positive solutions $u$ of (1) correspond to positive solutions $v$ of (3) and so $|v|^{8 /(n-4)} v=$ $v^{(n+4) /(n-4)}$. For some of our results, however, we also need to consider not necessarily positive functions $v$, and for such functions (3) is the relevant extension. We set

$$
a_{0}=\left(\frac{n(n-4)}{4}\right)^{(n-4) / 4} .
$$

Our first main result classifies all positive periodic solutions of (3) and describes their shape.

Theorem 1. (i) Let $v \in C^{4}(\mathbb{R})$ be a solution of (3). Then $\inf _{\mathbb{B}}|v| \leq a_{0}$, with equality if and only if $v$ is a nonzero constant.

(ii) Let $a \in\left(0, a_{0}\right)$. Then there is a unique (up to translations) bounded solution $v \in C^{4}(\mathbb{R})$ of (3) with minimal value a. This solution is periodic, has a unique local maximum and minimum per period and is symmetric with respect to its local extrema.

To state our second main result, we denote by $v_{a}$ the unique solution to (3) obtained from Theorem 1 by requiring that $v_{a}(0)=\min _{\mathbb{R}} v_{a}=a$. Also, denote by $L_{a}$ the minimal period of $v_{a}$. For the constant solution $v_{a_{0}} \equiv a_{0}$, we set $L_{a_{0}}=0$.

The following theorem provides a classification of positive solutions $u$ of (1) with nonremovable singularities in terms of a two-parameter family.

Theorem 2. Let $u \in C^{4}\left(\mathbb{R}^{n} \backslash\{0\}\right)$ be a positive solution of (1) whose singularity at the origin is nonremovable. Then there are $a \in\left(0, a_{0}\right]$ and $L \in\left[0, L_{a}\right]$ such that

$$
u(x)=|x|^{-(n-4) / 2} v_{a}(\log |x|+L),
$$

where $v_{a}$ is the solution of (3) introduced after Theorem 1. Moreover, $\partial u / \partial|x|<0$ for all $x \in \mathbb{R}^{n} \backslash\{0\}$.

This theorem answers an open question raised in [Guo et al. 2017a] and shows, in particular, that the positivity of the scalar curvature in their conjecture is not necessary. 
It is easy to see that as $a \rightarrow 0$ one has $L_{a} \rightarrow \infty$ and $v_{a}\left(t+L_{a} / 2\right) \rightarrow c_{n}(2 \cosh t)^{-(n-4) / 2}$. Undoing the logarithmic change of variables we therefore recover the nonsingular solution (2) in the limit $a \rightarrow 0$.

We believe that Theorems 1 and 2 will have several applications. Firstly, they should be a key step in describing the asymptotic behavior near the origin of positive solutions $u$ of $\Delta^{2} u=u^{(n+4) /(n-4)}$ in a punctured ball $\{0<|x|<\rho\}$. This would be the fourth-order analogue of a celebrated result of Caffarelli, Gidas and Spruck [Caffarelli et al. 1989]; see also [Korevaar et al. 1999]. Secondly, we believe that our theorems will prove useful in the construction of constant $Q$-curvature metrics with isolated singularities in the spirit of the classical works [Schoen 1988; Mazzeo and Pacard 1999] for the scalar curvature; see [Baraket and Rebhi 2002; Guo et al. 2017b] for results in this direction in the fourth-order case. For an introduction to the $Q$-curvature problem see, for instance, [Hang and Yang 2016].

We end this introduction by comparing the statements and proofs of Theorems 1 and 2 with their second-order counterpart, which concerns positive solutions $u$ of

$$
-\Delta u=u^{(n+2) /(n-2)} \quad \text { in } \mathbb{R}^{n} \backslash\{0\}
$$

for $n \geq 3$. A famous result of Caffarelli, Gidas and Spruck [Caffarelli et al. 1989] says that if this equation is valid on all of $\mathbb{R}^{n}$, then

$$
u(x)=c_{n}^{\prime}\left(\frac{\lambda}{1+\lambda^{2}\left|x-x_{0}\right|^{2}}\right)^{(n-2) / 2}, \quad c_{n}^{\prime}=(n(n-2))^{(n-2) / 4},
$$

for some $\lambda>0$ and $x_{0} \in \mathbb{R}^{n}$. Moreover, they show that if $u$ is a positive solution of (4) with a nonremovable singularity, then $u$ is radial. Using this information, Schoen [1989] observed that all solutions can be classified by standard phase-plane analysis. Indeed, setting

$$
u(x)=|x|^{-(n-2) / 2} v(\ln |x|)
$$

one obtains

$$
-v^{\prime \prime}+\frac{(n-2)^{2}}{4} v-v^{(n+2) /(n-2)}=0 \quad \text { in } \mathbb{R}
$$

and the positive solutions of this equation are given by the constant $((n-2) / 2)^{(n-2) / 2}$, by the homoclinic solution $c_{n}^{\prime}(2 \cosh (t+T))^{(n-2) / 2}$ and by periodic solutions uniquely parametrized, up to translations, by their minimal value in $\left(0,((n-2) / 2)^{(n-2) / 2}\right)$. Moreover, these periodic solutions have a unique local maximum and minimum per period and are symmetric with respect to their local extrema.

Thus, our Theorems 1 and 2 provide exactly the same conclusions as in the second-order case. Their proofs, however, are considerably more difficult, because the phase "plane" in the fourth-order case is four-dimensional. Moreover, solutions to fourth-order equations show, in general, a much richer and typically more erratic behavior than solutions to second-order equations; see, e.g., the introduction of the textbook [Peletier and Troy 2001] for examples. To emphasize the structure of our equation we abbreviate

$$
A=\frac{n(n-4)+8}{2}, \quad B=\frac{n^{2}(n-4)^{2}}{16}, \quad p=\frac{n+4}{n-4},
$$


and

$$
f(v)=|v|^{p-1} v-B v
$$

and rewrite (3) as

$$
v^{(4)}-A v^{\prime \prime}-f(v)=0 \quad \text { in } \mathbb{R} .
$$

Of fundamental importance for us is that the coefficients $A$ and $B$ in (3) satisfy the inequalities

$$
A>0 \text { and } 4 B<A^{2} .
$$

These inequalities guarantee that the characteristic equation $\xi^{4}-A \xi^{2}+B=0$ associated to the linearization of (7) around the zero solution has four distinct, real solutions. The picture that has emerged from the analysis of fourth-order equations is that under this structural assumption the solution set is better behaved than that of general fourth-order equations and resembles in some sense the solution set of second-order equations; see, e.g., [Peletier and Troy 2001; van den Berg 2000; Buffoni et al. 1996]. The reason is that certain techniques are available which are reminiscent of the maximum principle. Technically, this better, second-order-like behavior can be proved for bounded solutions of the equation and for such solutions there are certain substitutes for two-dimensional phase-plane arguments (see, in particular, Propositions 4 and 6). Parts of our analysis will rely on results of van den Berg [2000] for bounded solutions, which in turn rely on results of Buffoni, Champneys and Toland [Buffoni et al. 1996]. Our crucial new ingredient, however, which does not appear in these works, is that global solutions are necessarily bounded (Lemma 11). We emphasize that boundedness is a nonlocal property and breaks the local character of the ODE analysis.

Most of our results (except for the explicit expression of the homoclinic solution) hold, mutatis mutandis, for any equation of the form (7) with $f$ given by (6), where $p>1$ is arbitrary and $A$ and $B$ are arbitrary subject to (8).

\section{Classification of global ODE solutions}

In this section we will classify all solutions $v$ of (7) which are defined on all of $\mathbb{R}$. Positivity will not play a role here.

We begin with some preliminary remarks, which we will use several times below. The function $v \mapsto f(v)$ in (6) has exactly three zeros, namely, at 0 and at $\pm B^{1 /(p-1)}= \pm a_{0}$. These correspond to exactly three constant solutions. Moreover, if $v(t)$ is a solution to (7), then so are the functions

- $v(-t)$ (because (7) contains only even-order derivatives),

- $-v(t)$ (because $f$ is odd) and

- $v(t+T)$ for any $T \in \mathbb{R}$ (because (7) is autonomous).

We now state the main result of this section.

Proposition 3. Let $v \in C^{4}(\mathbb{R})$ be a solution of (7). Then one of the following three alternatives holds:

(a) $v \equiv \pm B^{1 /(p-1)}$, or $v \equiv 0$. 
(b) $v(t)= \pm c_{n}(2 \cosh (t-T))^{-(n-4) / 2}$ for some $T \in \mathbb{R}$ with $c_{n}$ from (2).

(c) $v$ is periodic, has a unique local maximum and minimum per period and is symmetric with respect to its local extrema.

For the proof of this proposition we will need two results, taken from [van den Berg 2000], which quantify the intuition that the set of bounded solutions to the fourth-order equation (7) behaves in some respects similarly to the set of solutions of a second-order equation. As we pointed out in the introduction, for this it is crucial that the relation $4 B<A^{2}$ is satisfied. The first result is that every bounded entire solution $v$ is uniquely determined by only two (instead of four) initial values.

Proposition 4 [van den Berg 2000, Theorem 1]. Let $v, w \in C^{4}(\mathbb{R})$ be bounded solutions of (7) and suppose that $v(0)=w(0)$ and $v^{\prime}(0)=w^{\prime}(0)$. Then $v \equiv w$.

Since this result is of crucial importance for us, we give a (slightly more direct) proof with our notation in the Appendix. Proposition 4 has the following consequence.

Corollary 5. Let $v \in C^{4}(\mathbb{R})$ be a bounded solution of $(7)$ :

(i) Suppose that $v^{\prime}\left(t_{0}\right)=0$ for some $t_{0} \in \mathbb{R}$. Then $v$ is symmetric with respect to $t_{0}$; i.e., for all $t \in \mathbb{R}$, $v\left(t_{0}+t\right)=v\left(t_{0}-t\right)$.

(ii) Suppose that $v\left(t_{0}\right)=0$ for some $t_{0} \in \mathbb{R}$. Then $v$ is antisymmetric with respect to $t_{0}$; i.e., for all $t \in \mathbb{R}$, $v\left(t_{0}-t\right)=-v\left(t_{0}+t\right)$.

Proof. (i) Since (7) is autonomous, we may assume $t_{0}=0$. Moreover, if $v$ is a solution, then so is $w(t):=v(-t)$. Thus $v(0)=w(0)$ and, by assumption, $v^{\prime}(0)=w^{\prime}(0)=0$. Proposition 4 gives $v \equiv w$.

(ii) Again, we may assume $t_{0}=0$. Moreover, if $v$ solves (7), then so does $w(t):=-v(-t)$. Since $v(0)=w(0)$ and $v^{\prime}(0)=w^{\prime}(0)$, we conclude by Proposition 4 that $v \equiv w$.

In order to state the second result from [van den Berg 2000] that we need, we introduce

$$
F(v)=\int_{0}^{v} f(s) \mathrm{d} s=\frac{|v|^{p+1}}{p+1}-\frac{1}{2} B v^{2}
$$

as well as the following quantity, also referred to as the energy:

$$
\mathcal{E}_{v}(t)=-v^{\prime \prime \prime}(t) v^{\prime}(t)+\frac{1}{2}\left(v^{\prime \prime}(t)\right)^{2}+\frac{1}{2} A\left(v^{\prime}(t)\right)^{2}+F(v(t)) .
$$

Using (7) one easily finds that for every solution $v$ of (7)

$$
\frac{\mathrm{d}}{\mathrm{d} t} \mathcal{E}_{v}(t)=0
$$

that is, the energy is conserved. We emphasize that this conservation is a local property and valid on the maximal interval of existence and does not require any a priori boundedness assumptions like Proposition 4 and the following Proposition 6 and Lemma 7.

The second result says that, as in the second-order case, the energy is a parameter which orders bounded solutions in the $\left(v, v^{\prime}\right)$-phase plane. 
Proposition 6 [van den Berg 2000, Theorem 2]. Let $v, w \in C^{4}(\mathbb{R})$ be bounded solutions of (7) with $v(0)=w(0)$ and either $v^{\prime}(0)>w^{\prime}(0) \geq 0$ or $v^{\prime}(0)<w^{\prime}(0) \leq 0$. Then $\mathcal{E}_{v}>\mathcal{E}_{w}$.

For the proof we refer to [van den Berg 2000]. The assumption there is satisfied since $4 B<A^{2}$. (Note that no a priori bound on the solutions is necessary for our $f$.)

Next, we state two lemmas concerning the asymptotic behavior of solutions at infinity.

Lemma 7 [van den Berg 2000, Lemma 4]. Let $v \in C^{4}(\mathbb{R})$ be a bounded solution of (7). If $v$ is eventually monotone for $t \rightarrow \infty$, then

$$
\lim _{t \rightarrow \infty} v(t) \in\left\{0, \pm B^{1 /(p-1)}\right\} \quad \text { and } \quad \lim _{t \rightarrow \infty} v^{(k)}(t)=0 \quad \text { for } k=1,2,3 .
$$

Similarly, if $v$ is eventually monotone for $t \rightarrow-\infty$, then

$$
\lim _{t \rightarrow-\infty} v(t) \in\left\{0, \pm B^{1 /(p-1)}\right\} \quad \text { and } \quad \lim _{t \rightarrow-\infty} v^{(k)}(t)=0 \quad \text { for } k=1,2,3 .
$$

The following lemma from [Guo et al. 2017a] shows that (7) does not have a solution which tends to either plus or minus infinity at infinity; that is, solutions that blow up do so in finite time.

Lemma 8 [Guo et al. 2017a, Lemma 2.1]. Let $v \in C^{4}(\mathbb{R})$ be a solution of (7). If $a_{+}:=\lim _{t \rightarrow \infty} v(t) \in$ $\mathbb{R} \cup\{ \pm \infty\}$ exists, then $a_{+} \in \mathbb{R}$. Similarly, if $a_{-}:=\lim _{t \rightarrow-\infty} v(t) \in \mathbb{R} \cup\{ \pm \infty\}$ exists, then $a_{-} \in \mathbb{R}$.

This lemma is proved in [Guo et al. 2017a] for positive solutions. An inspection of the proof shows, however, that this positivity is not needed.

We now use the above results to show uniqueness, up to translations, of the positive homoclinic solution. A similar result for $p=2$ appears in [Amick and Toland 1992] with a different proof.

Lemma 9. Let $v, w \in C^{4}(\mathbb{R})$ be positive solutions of (7) with $\lim _{|t| \rightarrow \infty} v(t)=\lim _{|t| \rightarrow \infty} w(t)=0$ and $v^{\prime}(0)=w^{\prime}(0)=0$. Then $v \equiv w$.

Proof. Let us first prove that 0 is the only zero of $v^{\prime}$ and $w^{\prime}$. Indeed, if $v^{\prime}$ had another zero at, say, $t_{0}>0$, then by repeated application of Corollary 5 (note that by assumption, $v$ is bounded) we deduce that $v$ must be periodic of period $2 t_{0}$. In particular $0<v(0)=v\left(2 k t_{0}\right)$ for all $k \in \mathbb{N}$, which contradicts the assumption that $v(t) \rightarrow 0$ as $t \rightarrow \infty$. The argument for $w$ is analogous. Hence we must have

$$
v^{\prime}(t)<0 \text { and } w^{\prime}(t)<0 \text { for all } t>0 .
$$

Next, by Lemma 7 and by energy conservation,

$$
\mathcal{E}_{v}=\lim _{t \rightarrow \infty} \mathcal{E}_{v}(t)=F(0)=0 \quad \text { and } \quad \mathcal{E}_{w}=\lim _{t \rightarrow \infty} \mathcal{E}_{w}(t)=F(0)=0
$$

If $v(0)=w(0)$, we are done by Proposition 4 .

To complete the proof, let us suppose for contradiction that $v(0)>w(0)$. We claim that this implies $v>w$ everywhere. Indeed, otherwise there is $t_{0}>0$ such that $v>w$ on $\left[0, t_{0}\right)$ and $v\left(t_{0}\right)=w\left(t_{0}\right)$. Then by (9) we infer that $v^{\prime}\left(t_{0}\right) \leq w^{\prime}\left(t_{0}\right)<0$. If $v^{\prime}\left(t_{0}\right)=w^{\prime}\left(t_{0}\right)$, then Proposition 4 implies $v \equiv w$, contradicting $v(0)>w(0)$. If $v^{\prime}\left(t_{0}\right)<w^{\prime}\left(t_{0}\right)<0$, then Proposition 6 implies $\mathcal{E}_{v}>\mathcal{E}_{w}$, which contradicts (10). Hence $v>w$ everywhere. 
We can now derive the desired contradiction. For every $R>0$, we have, using integration by parts and the fact that $v$ and $w$ satisfy (7),

$$
\begin{aligned}
0 & =\int_{-R}^{R} w\left(v^{(4)}-A v^{\prime \prime}-f(v)\right) \\
& =b(R)+\int_{-R}^{R} v\left(w^{(4)}-A w^{\prime \prime}-f(w)\right)+\int_{-R}^{R} w v\left(w^{p-1}-v^{p-1}\right) \\
& =b(R)+\int_{-R}^{R} w v\left(w^{p-1}-v^{p-1}\right) .
\end{aligned}
$$

Here, $b(R)$ contains all the boundary terms coming from the integrations by parts. By Lemma 7 we have $b(R) \rightarrow 0$ as $R \rightarrow \infty$. But since $\int_{-R}^{R} w v\left(w^{p-1}-v^{p-1}\right)$ is a negative and strictly decreasing function of $R$, we obtain a contradiction by choosing $R$ large enough.

For the concrete values of $A, B$ and $p$ in (5) one can compute the homoclinic solution explicitly. We emphasize that this is the only place in the proof of Proposition 3 where the precise form of $A, B$ and $p$ enters.

Corollary 10. Suppose that $v$ is a positive solution of (3) with $\lim _{|t| \rightarrow \infty} v(t)=0$. Then there is $T \in \mathbb{R}$ such that

$$
v(t)=c_{n}(2 \cosh (t-T))^{-(n-4) / 2}, \quad t \in \mathbb{R},
$$

with $c_{n}$ from (2).

Proof. A straightforward calculation shows that $w(t)=c_{n}(2 \cosh (t))^{-(n-4) / 2}$ solves (3). From the assumptions on $v$ it follows that $v$ has a global maximum at some $T \in \mathbb{R}$. Since $v^{\prime}(T)=0$, we can apply Lemma 9 to deduce that $v(\cdot+T)=w$.

The following lemma is one of the key new results in this paper.

Lemma 11. Let $v \in C^{4}(\mathbb{R})$ be a solution of (7). Then $v$ is bounded.

Proof. By replacing $v(t)$ by $v(-t)$, we only need to show that $v$ is bounded on $[0, \infty)$. We consider the set $Z_{+}=\left\{t \geq 0: v^{\prime}(t)=0\right\}$.

If $Z_{+}$is bounded (in particular, if it is empty), then $v$ is monotone for large $t$ and thus admits a limit $a_{+}$ as $t \rightarrow \infty$. By Lemma $8, a_{+}$is finite and therefore $v$ is bounded on $[0, \infty)$.

We now assume that $Z_{+}$is unbounded. Since $F(u) \rightarrow \infty$ as $|u| \rightarrow \infty$, there is an $R>|v(0)|$ such that $F(u)>\mathcal{E}_{v}$ for all $|u| \geq R$. We claim that $|v|<R$ on $[0, \infty)$ which, in particular, implies that $v$ is bounded on $[0, \infty)$. Indeed, by contradiction assume that $M_{R}:=\{t \geq 0:|v(t)| \geq R\}$ is nonempty and define $t^{*}:=\inf M_{R}$. Since $|v(0)|<R$, we must have $t^{*}>0$ and $\left|v\left(t^{*}\right)\right|=R$. Replacing $v(t)$ by $-v(t)$ if necessary (which does not change the set $Z_{+}$), we may assume that $v\left(t^{*}\right)=R$. Then also $v^{\prime}\left(t^{*}\right) \geq 0$. Since $Z_{+}$is unbounded, the set $Z_{+} \cap\left[t^{*}, \infty\right)$ is nonempty and we can set $T:=\inf \left(Z_{+} \cap\left[t^{*}, \infty\right)\right)$. Then $v^{\prime}(T)=0$ and $v^{\prime} \geq 0$ on $\left[t^{*}, T\right]$ by continuity of $v^{\prime}$. Thus $v(T) \geq v\left(t^{*}\right)=R$, and we deduce that

$$
\mathcal{E}_{v}(T)=\frac{1}{2} v^{\prime \prime}(T)^{2}+F(v(T)) \geq F(v(T))>\mathcal{E}_{v},
$$

a contradiction to energy conservation. 
We are now ready to prove the main result of this section.

Proof of Proposition 3. Let $v \in C^{4}(\mathbb{R})$ be a solution to (7) and set

$$
Z:=\left\{t \in \mathbb{R}: v^{\prime}(t)=0\right\} .
$$

We distinguish several cases:

Suppose first that $Z=\varnothing$, so $v$ is strictly monotone. We will show that this case cannot occur. Up to replacing $v(t)$ by $v(-t)$, we may assume that $v$ is strictly increasing, and so both limits $a_{ \pm}=\lim _{t \rightarrow \pm \infty} v(t)$ exist in $\mathbb{R} \cup\{ \pm \infty\}$. By Lemma 8 both limits are finite. By Lemma 7, we are reduced to studying three cases, each of which will lead to a contradiction via an energy argument.

If $a_{-}=0$ and $a_{+}=B^{1 /(p-1)}$, then using Lemma 7 we get $\lim _{t \rightarrow-\infty} \mathcal{E}_{v}(t)=F(0)=0$, while $\lim _{t \rightarrow+\infty} \mathcal{E}_{v}(t)=F\left(B^{1 /(p-1)}\right)<0$, a contradiction to energy conservation. Analogously, a contradiction is obtained if $a_{-}=-B^{1 /(p-1)}$ and $a_{+}=0$.

It remains to consider the case $a_{-}=-B^{1 /(p-1)}$ and $a_{+}=B^{1 /(p-1)}$. Then as above, by Lemma 7 ,

$$
\lim _{|t| \rightarrow \infty} \mathcal{E}_{v}(t)=F\left(B^{1 /(p-1)}\right)<0 .
$$

On the other hand, by [van den Berg 2000, Corollary 6], the inequality

$$
\mathcal{E}_{v}(t) \geq \frac{1}{2} v^{\prime \prime}(t)^{2}+F(v(t))
$$

holds for all $t \in \mathbb{R}$. But now evaluating the energy at $t_{0}$ such that $v\left(t_{0}\right)=0$ gives, together with (12), that $\mathcal{E}_{v}\left(t_{0}\right) \geq \frac{1}{2} v^{\prime \prime}\left(t_{0}\right)^{2}+F(0) \geq 0$, in contradiction to (11) and energy conservation. Altogether, we have shown that the case $Z=\varnothing$ cannot occur.

If $|Z|=1$, we may assume, up to a translation, that $Z=\{0\}$. Then $v$ is strictly monotone on $(-\infty, 0)$ and $(0, \infty)$, and so both limits $a_{ \pm}=\lim _{t \rightarrow \pm \infty} v(t)$ exist in $\mathbb{R} \cup\{ \pm \infty\}$. By Lemma 8 these limits are finite, so $v$ is bounded and, by Corollary 5, even. Therefore $a_{+}=a_{-}$. By Lemma 7, only three cases can occur: $a_{+}=a_{-}=0$ or $a_{+}=a_{-}= \pm B^{1 /(p-1)}$. In the first case, monotonicity implies that either $v>0$ or $v<0$, and we conclude that $v(t)= \pm c_{n}(2 \cosh (t))^{-(n-4) / 2}$ by Corollary 10 .

As for the other cases, let us assume without loss of generality that $a_{+}=a_{-}=B^{1 /(p-1)}$ (otherwise replace $v$ by $-v)$. We derive a contradiction as follows. Since $v$ is strictly monotone on $[0, \infty)$, $v(0) \neq B^{1 /(p-1)}$, and from $\mathcal{E}_{v}(0)=\frac{1}{2} v^{\prime \prime}(0)^{2}+F(v(0)) \geq F\left(B^{1 /(p-1)}\right)$ we infer that $v(0)=-B^{1 /(p-1)}$ ( since $F$ attains its global minimal value only at $\left.\pm B^{1 /(p-1)}\right)$. Hence $v$ changes sign; i.e., there is $t_{0} \in \mathbb{R}$ such that $v\left(t_{0}\right)=0$. By Corollary $5, v$ is antisymmetric with respect to $t_{0}$. But this is a contradiction to the fact that both $a_{+}$and $a_{-}$are positive. Altogether we have thus shown that if $|Z|=1$, then $v(t)= \pm c_{n}(2 \cosh (t))^{-(n-4) / 2}$.

Finally, let us consider the case where $|Z| \geq 2$. By continuity of $v^{\prime}$, we see that unless $v$ is constant (and hence $v \equiv \pm B^{1 /(p-1)}$ or $\left.v \equiv 0\right)$, the closed set $Z$ cannot be dense; i.e., there are real numbers $c<d$ such that $v^{\prime}(c)=v^{\prime}(d)=0$ and $v^{\prime} \neq 0$ on $(c, d)$. By Lemma $11, v$ is bounded and therefore we can use Corollary 5 as in the first part of the proof of Lemma 9 to conclude that $v$ must be periodic of period $2(d-c)$. Moreover, since $v$ is strictly monotone on $(c, d)$, there is only one maximum and minimum per period interval, and these are strict. The symmetry with respect to the extrema follows at once from Corollary 5. 
We end this section with one more result that will be needed in the proof of Theorem 2 .

Lemma 12. Let $v \in C^{4}(\mathbb{R})$ be a positive solution of (3). Then

$$
v^{\prime}<\sqrt{\frac{1}{2} A-\sqrt{\left(\frac{1}{2} A\right)^{2}-B}} v .
$$

For our values of $A$ and $B$ we have

$$
\sqrt{\frac{1}{2} A-\sqrt{\left(\frac{1}{2} A\right)^{2}-B}}=\frac{1}{2}(n-4)
$$

but the lemma is true for general $A$ and $B$ satisfying (8).

Proof. Because of (8) we can introduce the two positive numbers

$$
\lambda=\frac{1}{2} A-\sqrt{\left(\frac{1}{2} A\right)^{2}-B} \text { and } \mu=\frac{1}{2} A+\sqrt{\left(\frac{1}{2} A\right)^{2}-B} .
$$

Using $\lambda+\mu=A$ and $\lambda \mu=B$ we can write (3) in terms of the auxiliary function

$$
\phi(t):=v^{\prime \prime}(t)-\lambda v(t)
$$

as

$$
\phi^{\prime \prime}-\mu \phi=v^{p}
$$

According to Proposition 3, $\phi$ attains its maximum on $\mathbb{R}$. Since $v>0$, the maximum principle implies that $\phi<0$.

The function $w:=v^{\prime} / v$ satisfies

$$
w^{\prime}=-w^{2}+\lambda+\frac{\phi}{v}
$$

According to Proposition 3 there is a $t_{0} \in \mathbb{R}$ with $v^{\prime}\left(t_{0}\right)=0$, and therefore also $w\left(t_{0}\right)=0$. We shall show now that $M:=\left\{t>t_{0}: w(t) \geq \sqrt{\lambda}\right\}$ is empty, which yields the claimed inequality.

Suppose by contradiction that $M \neq \varnothing$ and let $t_{1}:=\inf M$. It is easy to see that $t_{1}>t_{0}$. Then certainly $w^{\prime}\left(t_{1}\right) \geq 0$. On the other hand, since $w\left(t_{1}\right)=\sqrt{\lambda}$, (15) implies

$$
w^{\prime}\left(t_{1}\right)=\frac{\phi\left(t_{1}\right)}{v\left(t_{1}\right)}<0,
$$

where the inequality comes from $\phi<0$ and $v>0$. This is a contradiction.

\section{Proofs of the main results}

3.1. Proof of Theorem 1. We begin with the proof of part (i) of Theorem 1. Let $v \in C^{4}(\mathbb{R})$ be a solution of (7). By Proposition 3, the only case where

$$
\inf _{\mathbb{R}}|v| \leq B^{1 /(p-1)}
$$

may fail to hold is when $v$ is periodic. In this case, $v$ possesses a local minimum at, say, $t_{0} \in \mathbb{R}$. Note that if $v$ has a zero then (16) is automatically fulfilled, so we may assume that $v$ has a fixed sign and, 
up to replacing $v$ by $-v$, we may assume that $v>0$. But by [Guo et al. 2017a, Lemma 2.6], either $v$ is constant (and hence $v \equiv B^{1 /(p-1)}$ ) or $v\left(t_{0}\right)<B^{1 /(p-1)}$, so that (16) holds with strict inequality.

We turn now to proving part (ii) of Theorem 1 . We proceed via a shooting argument. The value $a \in\left(0, B^{1 /(p-1)}\right)$ will be considered to be fixed throughout the following argument.

For $\beta \geq 0$, we denote by $v_{\beta}$ the unique solution of (7) with the initial values

$$
v(0)=a, \quad v^{\prime}(0)=0, \quad v^{\prime \prime}(0)=\beta, \quad v^{\prime \prime \prime}(0)=0,
$$

and by $T_{\beta} \in(0, \infty]$ its maximal forward time of existence. Also, let $b:=-\min _{v \in \mathbb{R}_{+}} f(v)$.

Suppose that $\beta>b / A=: \beta_{0}$. Then we see from

$$
v_{\beta}^{(4)}=A v_{\beta}^{\prime \prime}+f\left(v_{\beta}\right)
$$

and (17) that $v_{\beta}^{(4)}>0$ initially. Thus, $v_{\beta}^{\prime \prime}$ increases initially, and since the right-hand side of (18) is positive initially, it is easy to see that it will stay positive on $\left[0, T_{\beta}\right)$. Thus, $v_{\beta}^{(4)}>0$ on $\left[0, T_{\beta}\right)$, which implies that $v_{\beta}$ and its first three derivatives all keep increasing on $\left[0, T_{\beta}\right)$. Thus, if $T_{\beta}=\infty$, then $v_{\beta}$ is unbounded. On the other hand, if $T_{\beta}<\infty$, then $v_{\beta}(t) \rightarrow \infty$ as $t \rightarrow T_{\beta}$ (since $f$ is locally Lipschitz). To summarize, $v_{\beta}$ increases monotonically on $\left[0, T_{\beta}\right.$ ) and diverges to $+\infty$ as $t \rightarrow T_{\beta}$ for $\beta \geq \beta_{0}$.

So we can restrict our search to $\beta \in\left[0, \beta_{0}\right]$. However, for all $\beta \leq \beta_{0}$, we have the uniform energy bound

$$
\mathcal{E}_{v_{\beta}}(0)=\frac{1}{2} \beta^{2}+F(a) \leq \frac{1}{2} \beta_{0}^{2}+F(a) .
$$

Since $F(v) \rightarrow \infty$ as $v \rightarrow \infty$, there is an $R>0$ such that $F(v)>\frac{1}{2} \beta_{0}^{2}+F(a)$ for all $v>R$. This implies that whenever $\beta \leq \beta_{0}$ and $v_{\beta}\left(t_{0}\right)>R$, we must have $v_{\beta}^{\prime}\left(t_{0}\right) \neq 0$, for otherwise

$$
\mathcal{E}_{v_{\beta}}\left(t_{0}\right)=\frac{1}{2} v_{\beta}^{\prime \prime}\left(t_{0}\right)^{2}+F\left(v_{\beta}\left(t_{0}\right)\right) \geq F\left(v_{\beta}\left(t_{0}\right)\right)>\frac{1}{2} \beta_{0}^{2}+F(a),
$$

which contradicts the upper bound on $\mathcal{E}_{v_{\beta}}(0)$ and energy conservation. In particular, a $v_{\beta}$ which enters the interval $(R, \infty)$ cannot leave it again, and hence is certainly not the periodic solution we are looking for.

On the other hand, if $\beta=0$, we see from (18) that $v_{0}^{(4)}(0)=f(a)<0$, and hence $v_{0}(t)$ and $v_{0}^{\prime \prime}(t)$ are strictly decreasing on some small interval $t \in(0, \sigma)$. Since $f(v)<0$ for $v \in(0, a)$, we deduce from (18) that $v_{0}^{(k)}(t), k=1,2,3$, stay strictly negative until $v_{0}(t)$ reaches a negative value. Hence, if $\beta=0$, there must be $t_{0}$ such that $v_{0}\left(t_{0}\right)<0$.

All of the previous considerations lead us to defining the shooting sets

$$
\begin{aligned}
& S:=\left\{\beta \geq 0: v_{\beta}(t)<0 \text { for some } t \in\left(0, T_{\beta}\right)\right\}, \\
& T:=\left\{\beta \geq 0: v_{\beta}(t)>R \text { for some } t \in\left(0, T_{\beta}\right) \text { and } v_{\beta}>0 \text { on }[0, t]\right\} .
\end{aligned}
$$

Clearly, $S$ and $T$ are open in $[0, \infty)$ because of the continuous dependence of the solution on the initial conditions. Moreover, $S$ and $T$ are disjoint because, as we observed above, once a solution $v_{\beta}$ enters the interval $(R, \infty)$, it stays there. We also already argued above that $0 \in S$ and $\left(\beta_{0}, \infty\right) \subset T$; i.e., both $S \neq \varnothing$ and $T \neq \varnothing$.

Since our shooting parameter interval $[0, \infty)$ is connected, we deduce that $S \cup T \neq[0, \infty)$. Hence there must be $\beta^{*}>0$ and a corresponding solution $v^{*}:=v_{\beta^{*}}$ such that $0 \leq v^{*} \leq R$. In particular, $v^{*}$ is 
bounded. This and the fact that $f$ is locally Lipschitz imply that $T_{\beta^{*}}=\infty$. By even reflection, we obtain a solution defined on all of $\mathbb{R}$, which we still refer to as $v^{*}$. Since $\beta^{*}>0$, we know $v^{*}$ has a strict local minimum in 0 . By the classification of solutions from Proposition 3, $v^{*}$ must be periodic. Moreover, it has a unique local maximum and minimum per period and is symmetric with respect to its extrema.

The uniqueness of $v^{*}$ up to translations follows from Proposition 4.

3.2. Proof of Theorem 2. By [Lin 1998, Theorem 4.2], the positivity of $u$ and the nonremovability of the singularity in 0 imply that $u$ is radially symmetric. Since the function $v$ defined by $u(x)=$ $|x|^{-(n-4) / 2} v(\ln |x|)$ satisfies (3), we are in a position to apply the classification result from Proposition 3 and we claim that $v$ is either the constant $B^{1 /(p-1)}=a_{0}$ or periodic. Indeed, the only case that remains to be excluded is that $v(t)=c_{n}(2 \cosh (t-T))^{-(n-4) / 2}$. But in this case, it is clear that $v(t) \sim c_{n} e^{t(n-4) / 2}$ as $t \rightarrow-\infty$ and hence the singularity of $u$ would be removable, contradicting the assumptions. Thus, either $v$ is constant or periodic.

Let $a:=\inf v$. Then, by the first part of Theorem 1, $a \in\left(0, a_{0}\right]$, and $a=a_{0}$ if and only if $v \equiv a_{0}$. Moreover, for $a<a_{0}$ the function $v$ is periodic with minimal value $a$. Therefore, by the second part of Theorem $1, v(t)=v_{a}(t+L)$ for some $L \in \mathbb{R}$.

Finally, a simple computation shows that the inequality $\partial u / \partial|x|<0$ is equivalent to $v^{\prime}<\frac{1}{2}(n-4) v$, which follows from Lemma 12.

\section{Appendix: Proof of Proposition 4}

In this appendix, we give the proof of Proposition 4, following and simplifying [van den Berg 2000].

Let $v$ and $w$ be bounded solutions of (7) which satisfy $v(0)=w(0)$ and $v^{\prime}(0)=w^{\prime}(0)$. We can assume without loss that $v^{\prime \prime}(0) \geq w^{\prime \prime}(0)$ (otherwise exchange $v$ and $w$ ). We may assume furthermore (up to replacing $v(t)$ and $w(t)$ by $v(-t)$ and $w(-t))$ that $v^{\prime \prime \prime}(0) \geq w^{\prime \prime \prime}(0)$.

Suppose, by contradiction, that $v \neq \equiv w$. Then by uniqueness of ODE solutions, $v^{(k)}(0) \neq w^{(k)}(0)$ for $k=2$ or $k=3$. In both cases, we deduce from our hypotheses on the initial conditions that

$$
v(t)>w(t) \quad \text { on }(0, \sigma)
$$

for some sufficiently small $\sigma>0$.

With the positive numbers $\lambda$ and $\mu$ from (13) we define the auxiliary functions

$$
\phi(t):=v^{\prime \prime}(t)-\lambda v(t) \quad \text { and } \quad \psi(t):=w^{\prime \prime}(t)-\lambda w(t) .
$$

Then by the hypotheses, we have

$$
(\phi-\psi)(0) \geq 0 \quad \text { and } \quad(\phi-\psi)^{\prime}(0) \geq 0 .
$$

As in (14), equation (7) for $v$ and $w$ implies

$$
(\phi-\psi)^{\prime \prime}(t)-\mu(\phi-\psi)(t)=|v(t)|^{p-1} v(t)-|w(t)|^{p-1} w(t) \quad \text { for all } t \in \mathbb{R} .
$$


Since $v(t)>w(t)$ on $(0, \sigma)$ and since the function $u \mapsto|u|^{p-1} u$ is strictly increasing on $\mathbb{R}$, this implies

$$
(\phi-\psi)^{\prime \prime}(t)-\mu(\phi-\psi)(t)>0 \text { for all } t \in(0, \sigma) .
$$

The inequalities (19) and (20) and the fact that $\mu>0$ easily imply $(\phi-\psi)(t) \geq 0$ for $t \in(0, \sigma)$, or equivalently,

$$
(v-w)^{\prime \prime}(t) \geq \lambda(v-w)(t)>0 \quad \text { for all } t \in(0, \sigma) .
$$

Since $(v-w)^{\prime}(0) \geq 0$ by the hypotheses of the lemma and since $\lambda>0$, we see from $(21)$ that $(v-w)^{\prime}(t)>0$ for all $t \in(0, \sigma)$. Hence $v-w$ is strictly increasing on $(0, \sigma)$ and since $\sigma>0$ was arbitrary with the property that $v-w>0$ on $(0, \sigma)$, we infer that $v-w$ remains strictly positive for all times.

Repeating the above arguments for the interval $(0, \infty)$ instead of $(0, \sigma)$, we see from $(21)$ that $(v-w)^{\prime}$ is positive and strictly increasing on $(0, \infty)$. This of course contradicts the boundedness of $v-w$. This proves that in fact we must have $v \equiv w$, concluding the proof of Proposition 4.

\section{References}

[Amick and Toland 1992] C. J. Amick and J. F. Toland, "Homoclinic orbits in the dynamic phase-space analogy of an elastic strut”, European J. Appl. Math. 3:2 (1992), 97-114. MR Zbl

[Baraket and Rebhi 2002] S. Baraket and S. Rebhi, "Construction of dipole type singular solutions for a biharmonic equation with critical Sobolev exponent", Adv. Nonlinear Stud. 2:4 (2002), 459-476. MR Zbl

[van den Berg 2000] J. B. van den Berg, "The phase-plane picture for a class of fourth-order conservative differential equations", J. Differential Equations 161:1 (2000), 110-153. MR Zbl

[Buffoni et al. 1996] B. Buffoni, A. R. Champneys, and J. F. Toland, "Bifurcation and coalescence of a plethora of homoclinic orbits for a Hamiltonian system", J. Dynam. Differential Equations 8:2 (1996), 221-279. MR Zbl

[Caffarelli et al. 1989] L. A. Caffarelli, B. Gidas, and J. Spruck, "Asymptotic symmetry and local behavior of semilinear elliptic equations with critical Sobolev growth", Comm. Pure Appl. Math. 42:3 (1989), 271-297. MR Zbl

[Chang and Yang 1997] S.-Y. A. Chang and P. C. Yang, "On uniqueness of solutions of $n$th order differential equations in conformal geometry", Math. Res. Lett. 4:1 (1997), 91-102. MR Zbl

[Chang et al. 1999] S.-Y. A. Chang, M. J. Gursky, and P. C. Yang, "Regularity of a fourth order nonlinear PDE with critical exponent", Amer. J. Math. 121:2 (1999), 215-257. MR Zbl

[Guo et al. 2017a] Z. Guo, X. Huang, L. Wang, and J. Wei, "On Delaunay solutions of a biharmonic elliptic equation with critical exponent", preprint, 2017. arXiv

[Guo et al. 2017b] Z. Guo, J. Wei, and F. Zhou, "Singular radial entire solutions and weak solutions with prescribed singular set for a biharmonic equation", J. Differential Equations 263:2 (2017), 1188-1224. MR Zbl

[Hang and Yang 2016] F. Hang and P. C. Yang, "Lectures on the fourth-order $Q$ curvature equation", pp. 1-33 in Geometric analysis around scalar curvatures, edited by F. Han et al., Lect. Notes Ser. Inst. Math. Sci. Natl. Univ. Singap. 31, World Scientific, Hackensack, NJ, 2016. MR Zbl

[Korevaar et al. 1999] N. Korevaar, R. Mazzeo, F. Pacard, and R. Schoen, "Refined asymptotics for constant scalar curvature metrics with isolated singularities", Invent. Math. 135:2 (1999), 233-272. MR Zbl

[Lieb 1983] E. H. Lieb, "Sharp constants in the Hardy-Littlewood-Sobolev and related inequalities", Ann. of Math. (2) 118:2 (1983), 349-374. MR Zbl

[Lin 1998] C.-S. Lin, "A classification of solutions of a conformally invariant fourth order equation in $\mathbb{R}^{n}$ ", Comment. Math. Helv. 73:2 (1998), 206-231. MR Zbl

[Martinazzi 2009] L. Martinazzi, "Classification of solutions to the higher order Liouville's equation on $\mathbb{R}^{2 m}$ ", Math. Z. 263:2 (2009), 307-329. MR Zbl 
[Mazzeo and Pacard 1999] R. Mazzeo and F. Pacard, "Constant scalar curvature metrics with isolated singularities", Duke Math. J. 99:3 (1999), 353-418. MR Zbl

[Peletier and Troy 2001] L. A. Peletier and W. C. Troy, Spatial patterns: higher order models in physics and mechanics, Progress in Nonlinear Differential Equations and their Applications 45, Birkhäuser, Boston, 2001. MR Zbl

[Schoen 1988] R. M. Schoen, "The existence of weak solutions with prescribed singular behavior for a conformally invariant scalar equation", Comm. Pure Appl. Math. 41:3 (1988), 317-392. MR Zbl

[Schoen 1989] R. M. Schoen, "Variational theory for the total scalar curvature functional for Riemannian metrics and related topics", pp. 120-154 in Topics in calculus of variations (Montecatini Terme, 1987), edited by M. Giaquinta, Lecture Notes in Math. 1365, Springer, 1989. MR Zbl

[Uhlenbeck and Viaclovsky 2000] K. K. Uhlenbeck and J. A. Viaclovsky, "Regularity of weak solutions to critical exponent variational equations", Math. Res. Lett. 7:5-6 (2000), 651-656. MR Zbl

[Wei and Xu 1999] J. Wei and X. Xu, "Classification of solutions of higher order conformally invariant equations", Math. Ann. 313:2 (1999), 207-228. MR Zbl

Received 2 Nov 2017. Revised 28 May 2018. Accepted 30 Jul 2018.

RUPERT L. FRANK: r.frank@lmu.de, rlfrank@caltech.edu

Mathematisches Institut, Ludwig-Maximilians-Universität München, München, Germany

and

Department of Mathematics, Caltech, Pasadena, CA, United States

TOBIAS KÖNIG: tkoenig@math.lmu.de

Mathematisches Institut, Ludwig-Maximilians-Universität München, München, Germany 


\section{Analysis \& PDE}

msp.org/apde

\section{EDITORS}

EDITOR-IN-CHIEF

Patrick Gérard

patrick.gerard@math.u-psud.fr

Université Paris Sud XI

Orsay, France

BOARD OF EDITORS
Clément Mouhot

Werner Müller

Gilles Pisier

Tristan Rivière

Igor Rodnianski

Sylvia Serfaty

Yum-Tong Siu

Terence Tao

Michael E. Taylor

Gunther Uhlmann

András Vasy

Dan Virgil Voiculescu

Steven Zelditch

Maciej Zworski
Cambridge University, UK c.mouhot@dpmms.cam.ac.uk

Universität Bonn, Germany mueller@math.uni-bonn.de

Texas A\&M University, and Paris 6 pisier@math.tamu.edu

ETH, Switzerland riviere@math.ethz.ch

Princeton University, USA irod@math.princeton.edu

New York University, USA serfaty@cims.nyu.edu

Harvard University, USA siu@math.harvard.edu

University of California, Los Angeles, USA tao@math.ucla.edu

Univ. of North Carolina, Chapel Hill, USA met@math.unc.edu

University of Washington, USA gunther@math.washington.edu

Stanford University, USA andras@math.stanford.edu

University of California, Berkeley, USA dvv@math.berkeley.edu

Northwestern University, USA zelditch@math.northwestern.edu

University of California, Berkeley, USA zworski@math.berkeley.edu

PRODUCTION

production@msp.org

Silvio Levy, Scientific Editor

See inside back cover or msp.org/apde for submission instructions.

The subscription price for 2019 is US $\$ 310 /$ year for the electronic version, and $\$ 520 /$ year (+\$60, if shipping outside the US) for print and electronic. Subscriptions, requests for back issues from the last three years and changes of subscriber address should be sent to MSP.

Analysis \& PDE (ISSN 1948-206X electronic, 2157-5045 printed) at Mathematical Sciences Publishers, 798 Evans Hall \#3840, c/o University of California, Berkeley, CA 94720-3840, is published continuously online. Periodical rate postage paid at Berkeley, CA 94704, and additional mailing offices.

APDE peer review and production are managed by EditFlow ${ }^{\circledR}$ from MSP.

PUBLISHED BY

mathematical sciences publishers

nonprofit scientific publishing

http://msp.org/

(C) 2019 Mathematical Sciences Publishers 


\section{ANALYSIS \& PDE}

\section{Volume $12 \quad$ No. $4 \quad 2019$}

Quantum dynamical bounds for ergodic potentials with underlying dynamics of zero topolog- 867 ical entropy

RUi HAN and SVETLANA JiTOMIRSKAYA

Two-dimensional gravity water waves with constant vorticity, I: Cubic lifespan

MIHAELA IFRIM and DANIEL TATARU

Absolute continuity and $\alpha$-numbers on the real line

TUOMAS ORPONEN

Global well-posedness for the two-dimensional Muskat problem with slope less than 1

997 STEPHEN CAMERON

Global well-posedness and scattering for the radial, defocusing, cubic wave equation with 1023 initial data in a critical Besov space BENJAMIN DODSON

Nonexistence of Wente's $L^{\infty}$ estimate for the Neumann problem JONAS HIRSCH

Global geometry and $C^{1}$ convex extensions of 1-jets 1065

DANIEL AZAGRA and CARLOS MUdARRA

Classification of positive singular solutions to a nonlinear biharmonic equation with critical 1101 exponent

RUPERT L. FRANK and TOBIAS KÖNIG

Optimal multilinear restriction estimates for a class of hypersurfaces with curvature 